\title{
Inferior vena cava distensibility index predicting fluid responsiveness in ventilated patients
}

\author{
J Lujan Varas", C Martinez Díaz, R Blancas, O Martinez Gonzalez, B Llorente Ruiz, R Molina Montero, \\ C Arenillas Juanas, A Pardo, L Alcázar Sánchez-Elvira, JA Cambronero Galache \\ From ESICM LIVES 2015 \\ Berlin, Germany. 3-7 October 2015
}

\section{Introduction}

Echocardiography is a non-invasive procedure which enables full assesment of cardiac function. The inferior vena cava (IVC) is a compliant blood vessel, easily distended, especially in cases of hypovolemia. Assessment of the physiologic characteristics of the IVC provides a rapid distinction between low and high volume states and offers the clinician a rapid, noninvasive way to guide resuscitation in critically ill patients.

\section{Objectives}

To assess the reliability of the distensibility of inferior vena cava (dIVC), measured by ultrasound, as an indicator of fluid responsiveness in ventilated patients

\section{Methods}

Observational prospective study in a 14-bed Intensive Care Unit. We enrolled 15 patients requiring advanced hemodynamic monitoring (PiCCO, Vigileo, Swan-Ganz catheter). The dIVC was calculate as (maximum diameter - minimum diameter)/minimum diameter and possible responders were defined as dIVC $>18 \%$.IVC assessment was done just proximal to the hepatic veins, which lie approximately 0.5 to $3 \mathrm{~cm}$ from the right atrium, following the American Society of Echocardiography guidelines. Hemodynamic parameters were collected at baseline and after a fluid challenge. Fluid challenge was made by de maneuver of passive legs raising (PLR) that mimics a fluid challenge of $300 \mathrm{ml}$. Fluid responsiveness was defined by an increase of $>15 \%$ in cardiac output. Demographics characteristics, reason of ICU admission, severity of illness by APACHE and necessity of vasopressor support were also collected.

Hospital Principe de Asturias, Madrid, Spain

\section{Results}

We included 15 patients with an age mean of $64.67+14.1$, $40 \%$ male. Abdominal septic shock was the most frequent reason of ICU admission(40\%),respiratory (20\%),cardiogenic (13.3\%) and others(20\%).Median APACHE was $19.27+5.86$. All patients were on mechanical ventilation with PEEP mean $11.40+3.74$. All patients were on sensual rhythm and $80 \%$ needed vasopressor support. Advance hemodynamic monitoring was made by using PiCCO, Vigileo and Swan-Ganz catheter, $60 \%, 26.7 \%$ and $13.3 \%$, respectively. dIVC was $>18 \%$ in 4 patients $(26.7 \%)$ and 2 patients (50\%) responded to fluid challenge. In 11 patients (73.11\%) dIVC was < $18 \%$ and 10 of them (90.9\%) didn't respond to fluid challenge. Statistical analysis showed no significant differences $(\mathrm{p}>0.05)$.

\section{Conclusions}

Assessment of the IVC distensibility index in mechanically ventilated patients provides a useful and reliable tool in predicting response to volume in critically ill patients. Although our data do not show a statistical significance probably due to sample size, measuring the VCI should be part of a hemodynamic assessment specific protocol to evaluate the necessity or not of volume, that it is so important in the evolution of critical patients.

Published: 1 October 2015

\section{Reference}

1. Barbier C, Loubieres Y, Schmit C, Hayon J, Ricome JL, Jardin F, VieillardBaron A: Respiratory changes in inferior vena cava diameter are helpful in predicting fluid responsiveness in ventilated septic patients. Intensive Care Med 2004, 30(9):1740-1746.

\section{doi:10.1186/2197-425X-3-S1-A600}

Cite this article as: Lujan Varas et al:: Inferior vena cava distensibility index predicting fluid responsiveness in ventilated patients. Intensive Care Medicine Experimental 2015 3(Suppl 1):A600. 